\title{
Approximation of Isomorphic Infinite Two-Person Non-Cooperative Games by Variously Sampling the Players' Payoff Functions and Reshaping Payoff Matrices into Bimatrix Game
}

\author{
Vadim V. Romanuke ${ }^{1}$, Vladimir V. Kamburg ${ }^{2}$ \\ ${ }^{1}$ Khmelnitskiy National University, Ukraine, ${ }^{2}$ Penza State University of Architecture and Building, Russia
}

\begin{abstract}
Approximation in solving the infinite two-person non-cooperative games is studied in the paper. An approximation approach with conversion of infinite game into finite one is suggested. The conversion is fulfilled in three stages. Primarily the players' payoff functions are sampled variously according to the stated requirements to the sampling. These functions are defined on unit hypercube of the appropriate Euclidean finitedimensional space. The sampling step along each of hypercube dimensions is constant. At the second stage, the players' payoff multidimensional matrices are reshaped into ordinary twodimensional matrices, using the reversible index-to-index reshaping. Thus, a bimatrix game as an initial infinite game approximation is obtained. At the third stage of the conversion, the player's finite equilibrium strategy support is checked out for its weak consistency, defined by five types of inequalities within minimal neighbourhood of every specified sampling step. If necessary, the weakly consistent solution of the bimatrix game is checked out for its consistency, strengthened in that the cardinality of every player's equilibrium strategy support and their densities shall be non-decreasing within minimal neighbourhood of the sampling steps. Eventually, the consistent solution certifies the game approximation acceptability, letting solve even games without any equilibrium situations, including isomorphic ones to the unit hypercube game. A case of the consistency light check is stated for the completely mixed Nash equilibrium situation.
\end{abstract}

Keywords - Approximate solution, equilibrium strategy support, game isomorphism, multidimensional matrix, solution consistency, two-person non-cooperative games, unit hypercube.

\section{INFINITE TWO-PERSON NON-COOPERATIVE GAMES, ISOMORPHIC TO THE UNIT HYPERCUBE GAME}

There is always the question of resource distribution under everlasting demands and wants. The contemporary game theory grants a powerful logical apparatus for distributing resources fairly and profitably within equilibrium stability [1]. However, having the solution of a conflict event, which is modleled mathematically with a game, does not assign a way to implement it [2]. For instance, a Nash equilibrium strategy or other equilibrium type strategy with its finite support probability measure might have been practiced only with an infinite number of recurring support pure strategies [3], [4]. In the case of the infinite support probability measure, the majority of the support pure strategies cannot be recurred even once as a number of the recurred ones constitute the zero- measure set [5], [6]. However, for most classes of infinite twoperson non-cooperative games their solutions are unknown or at least are non-effectively computable [1], [2], [7]. Then, the conversion of the infiniteness into finiteness is needed anyway. This is approximation, i.e., conversion of an infinite two-person non-cooperative game into a finite one.

Two-person non-cooperative games model a series of conflict events, involving economic competition [1], [2], [8], [9], political controversy [1], [10], social discrepancy [11], [12], environmental engineering [13], ecological incompatibility [2], [14], jurisprudential confrontation [15], etc. In these events, the player, personifying a side of the conflict, often possesses an infinite set of pure strategies, usually being multiparametric. These sets, if constituting the compacts, predetermine non-cooperative compact games [2], [16], [17], which are isomorphic to the two-person non-cooperative game on a hyperparallelepiped of the appropriate Euclidean finitedimensional space (EFDS). Particularly, single-parametric pure strategies predetermine games on rectangles of $\mathbb{R}^{2}$. The simplest case of the hyperparallelepiped is a unit hypercube. Thus, for solving infinite two-person non-cooperative games with compact players' action spaces, it is sufficient [2], [18] to solve the two-person noncooperative game on a unit hypercube of the appropriate dimension.

\section{SOLVING THE TWO-PERSON NON-COOPERATIVE GAMES}

Exact methods of solving finite two-person non-cooperative games are based on linear programming or linear inequality manipulations, implying algorithms of Lemke - Howson [19], [20] or of Vorobyov [21] and Kuhn [22]. Such games can also be solved approximately [23]. Infinite games, if there are equilibrium strategies for both players, are solved in most cases approximately rather than exactly. Approximation of the game is a way of obtaining approximate equilibrium strategies of its players. Since it is much harder to implement the solution with infinite support, after approximation the players' equilibrium strategies have finite supports [5], [24], [25]. However, sometimes we cannot even find the solution, not mentioning the support finiteness. And just the approximation into the bimatrix game stands out the only route to solve.

Finding the infinite game solution approximately is sometimes ambiguous. Thus, one wants to use the theorem 
about existence of Nash equilibrium mixed strategies in the compact game [2] or to find $\varepsilon$-equilibrium strategies with finite supports, and the other one attempts to convert the game into the bimatrix game with applying subsequently a solver for it [19], [21], [23], [26]. Obviously that the second way is more preferable as once the bimatrix game is suggested then any of algorithms for solving it exactly may be applied, whereas solving directly the infinite game is not always possible, notably for greater dimensions of EFDS. Thus, it is better to approximate into the bimatrix game, which is solved much effectively rather than checking strategic approximation [24] for existence.

Another advantage of the game approximation through converting into the bimatrix game is that there is no universal technique for solving infinite two-person non-cooperative games [1], [2], [8], [16], [20], [27], [28]. Existence of Nash equilibrium solutions in compact games does not mean the existence of a technique to find one. However, solvers of bimatrix games of huge matrix format take large computational resources to get the exact solutions [23], [26]. However, more powerful cluster or cloud computers can accelerate the computational process even with non-square matrices [29]. Moreover, a particular class of bimatrix games with square payoff matrices is solved into completely mixed Nash equilibrium strategies just with the one statement [30], involving the inverse matrices of both players' payoffs, if they are nonsingular, though.

\section{GOAL AND OBJECTIVES OF THE RESEARCH}

In order to solve infinite two-person non-cooperative games, being isomorphic to the non-cooperative game on unit hypercube of EFDS, a method of the game approximation should be developed. For this reason, there are the following items to be fulfilled:

1. To define the players' payoff functions on unit hypercube of the appropriate EFDS.

2. To sample the players' payoff functions with specified sampling steps along every dimension of the unit hypercube.

3. To avoid removing distinctive features of the players' payoff functions in the sampling.

4. To reshape the players' multidimensional payoff matrices, approximating their payoff functions into twodimensional matrices.

5. To suggest conditions of its acceptance for the drawn bimatrix game, implying consistency of the player's equilibrium strategy support (ESS) under some form of utility equilibrium.

6. To discuss the acceptability of infinite two-person games approximation under possibilities for expanding the stated approximation concept.

\section{SAMPLING THE PLAYERS’ PAYOFF FUnCTIONS VARIOUSLY}

Let the $r$-th player's payoff function $K_{r}(\mathbf{X}, \mathbf{Y})$ be defined on $\left(M_{1}+M_{2}\right)$-dimensional unit hypercube

$$
\begin{aligned}
U_{M_{1}} \times U_{M_{2}} & =\left\{\mathbf{X}_{m_{1}=1}^{M_{1}}[0 ; 1]\right\} \times\left\{\mathbf{X}_{m_{2}=1}^{M_{2}}[0 ; 1]\right\}= \\
& =\left\{\chi_{m=1}^{M_{1}+M_{2}}[0 ; 1]\right\} \subset \mathbb{R}^{M_{1}+M_{2}}
\end{aligned}
$$

by

$$
\begin{gathered}
\mathbf{X}=\left[x_{m_{1}}\right]_{1 \times M_{1}} \in \chi_{m_{1}=1}^{M_{1}}[0 ; 1]=U_{M_{1}} \subset \mathbb{R}^{M_{1}} \text { and } \\
\mathbf{Y}=\left[y_{m_{2}}\right]_{1 \times M_{2}} \in \chi_{m_{2}=1}^{M_{2}}[0 ; 1]=U_{M_{2}} \subset \mathbb{R}^{M_{2}} \text { at }\left\{M_{r} \in \mathbb{N}\right\}_{r \in\{1,2\}},
\end{gathered}
$$

where the $r$-th player's pure strategy set is

$$
U_{M_{r}}=\bigcup_{m_{r}=1}^{M_{r}}[0 ; 1] \subset \mathbb{R}^{M_{r}} \text { for } r \in\{1,2\} .
$$

Functions $\left\{K_{1}(\mathbf{X}, \mathbf{Y}), K_{2}(\mathbf{X}, \mathbf{Y})\right\}$ are assumed to be bounded and measurable on (1). Hence, there is an infinite two-person non-cooperative game

$$
\left\langle U_{M_{1}}, U_{M_{2}}, K_{1}(\mathbf{X}, \mathbf{Y}), K_{2}(\mathbf{X}, \mathbf{Y})\right\rangle,
$$

which is isomorphic to any two-person non-cooperative game on a nonzero-measure hyperparallelepiped of $\mathbb{R}^{M_{1}+M_{2}}$ with the bounded and measurable functions of the players' payoffs, defined on this $\left(M_{1}+M_{2}\right)$-dimensional hyperparallelepiped.

Each of the functions $K_{1}(\mathbf{X}, \mathbf{Y})$ and $K_{2}(\mathbf{X}, \mathbf{Y})$ is presumed to be differentiable with respect to any of variables $\left\{\left\{x_{m_{1}}\right\}_{m_{1}=1}^{M_{1}},\left\{y_{m_{2}}\right\}_{m_{2}=1}^{M_{2}}\right\}$. There are also mixed derivatives of each of those functions by any combination of variables, where every variable is included no more than just once. These conditions are applied to requirements below, which must be followed to sample the players' payoff functions.

Let the function $K_{r}(\mathbf{X}, \mathbf{Y})$ be sampled along $\left(M_{1} \cdot \operatorname{sign}(r-1)+m_{r}\right)$-th dimension of hypercube (1) with the sampling step determined by the number $S_{r}\left(m_{r}\right)$ of intervals between the selected points in $\left(M_{1} \cdot \operatorname{sign}(r-1)+m_{r}\right)$-th dimension. It is conventional that endpoints of the unit segment $[0 ; 1]$ are included into the sampling necessarily, so $\left\{S_{r}\left(m_{r}\right) \in \mathbb{N}\right\}_{r \in\{1,2\}} \forall m_{r}=\overline{1, M_{r}}$. If we count the sampling step $\left[S_{r}\left(m_{r}\right)\right]^{-1}$ invariable along $\left(M_{1} \cdot \operatorname{sign}(r-1)+m_{r}\right)$-th dimension for $r \in\{1,2\}$ then in $m_{1}$-th dimension the first player instead of the segment $[0 ; 1]$ of values of $m_{1}$-th component of its pure strategy $\mathbf{X}$ acquires the set of points 


$$
\begin{gathered}
\left.D_{m_{1}}^{\langle\mathbf{X}\rangle}\left(S_{1}\left(m_{1}\right)\right)=\left\{x_{m_{1}}\right\}_{s=1}^{\langle\rangle\rangle}\right\}_{s=1}^{S_{1}\left(m_{1}\right)+1} \subset[0 ; 1] \text { by } \\
x_{m_{1}}^{\langle s\rangle}=\frac{s-1}{S_{1}\left(m_{1}\right)} \quad \forall m_{1}=\overline{1, M_{1}} .
\end{gathered}
$$

Simultaneously, in $m_{2}$-th dimension the second player instead of the segment $[0 ; 1]$ of values of $m_{2}$-th component of its pure strategy $\mathbf{Y}$ acquires the set of points

$$
\begin{gathered}
D_{m_{2}}^{\langle\mathbf{Y}\rangle}\left(S_{2}\left(m_{2}\right)\right)=\left\{y_{m_{2}}^{\langle s\rangle}\right\}_{s=1}^{S_{2}\left(m_{2}\right)+1} \subset[0 ; 1] \text { by } \\
y_{m_{2}}^{\langle s\rangle}=\frac{s-1}{S_{2}\left(m_{2}\right)} \quad \forall m_{2}=\overline{1, M_{2}} .
\end{gathered}
$$

Now the $r$-th player instead of the infinite set (2) possesses the finite set of its pure strategies, included $\prod_{m_{r}=1}^{M_{r}}\left(S_{r}\left(m_{r}\right)+1\right)$ points of hypercube (2). Consequently, infinite game (3) is substituted with the finite one

$$
\begin{gathered}
\left\langle\chi_{m_{1}=1}^{M_{1}} D_{m_{1}}^{\langle\mathbf{X}\rangle}\left(S_{1}\left(m_{1}\right)\right), \chi_{m_{2}=1}^{M_{2}} D_{m_{2}}^{\langle\mathbf{Y}\rangle}\left(S_{2}\left(m_{2}\right)\right)\right. \\
\left.K_{1}(\mathbf{X}, \mathbf{Y}), K_{2}(\mathbf{X}, \mathbf{Y})\right\rangle
\end{gathered}
$$

by

$$
\mathbf{X} \in \chi_{m_{1}=1}^{M_{1}} D_{m_{1}}^{\langle\mathbf{x}\rangle}\left(S_{1}\left(m_{1}\right)\right) \text { and } \mathbf{Y} \in \bigcup_{m_{2}=1}^{M_{2}} D_{m_{2}}^{\langle\mathbf{Y}\rangle}\left(S_{2}\left(m_{2}\right)\right)
$$

defined on the lattice

$$
\begin{gathered}
\left\{\chi_{m_{1}=1}^{M_{1}} D_{m_{1}}^{\langle\mathbf{X}\rangle}\left(S_{1}\left(m_{1}\right)\right)\right\} \times\left\{\chi_{m_{2}=1}^{M_{2}} D_{m_{2}}^{\langle\mathbf{Y}\rangle}\left(S_{2}\left(m_{2}\right)\right)\right\} \subset \\
\subset U_{M_{1}} \times U_{M_{2}} .
\end{gathered}
$$

However, now at $M_{1} \in \mathbb{N} \backslash\{1\}$ or $M_{2} \in \mathbb{N} \backslash\{1\}$ the game (6) by (7) still is not bimatrix inasmuch as the players' payoff values $K_{1}(\mathbf{X}, \mathbf{Y})$ and $K_{2}(\mathbf{X}, \mathbf{Y})$ by (7) are not arranged as ordinary flat matrices. The sampling on hypercube (1) down to lattice (8) with (4) and (5) is just the primary and incomplete step in converting the infinite game into the bimatrix game. Sampling the players' payoff functions variously due to distinct numbers

$$
\left\{\left\{S_{r}\left(m_{r}\right)\right\}_{m_{r}=1}^{M_{r}}\right\}_{r \in\{1,2\}}
$$

may also help in constructing square bimatrix games.

\section{REQUIREMENTS TO THE SAMPLING}

It is clear that numbers (9), defining the sampling steps

$$
\left\{\left\{\left[S_{r}\left(m_{r}\right)\right]^{-1}\right\}_{m_{r}=1}^{M_{r}}\right\}_{r \in\{1,2\}}
$$

to get the finite game (6) by (7), shall not be chosen incautiously. The sampling with steps (10) must not erase specificities of the players' payoff functions, particularly consisting in local extremums and gradient over hypersurfaces $\left\{K_{r}(\mathbf{X}, \mathbf{Y})\right\}_{r \in\{1,2\}}$. In sampling, the sampling steps (10) are required that $\forall s=\overline{1, S_{r}\left(m_{r}\right)+1}$ by $m_{r}=\overline{1, M_{r}}$ there would be

$$
\begin{gathered}
\frac{\partial^{M_{1}+M_{2}} K_{r}(\mathbf{X}, \mathbf{Y})}{\partial x_{1} \partial x_{2} \ldots \partial x_{M_{1}} \partial y_{1} \partial y_{2} \ldots \partial y_{M_{2}}} \geqslant 0 \quad \text { or } \\
\frac{\partial^{M_{1}+M_{2}} K_{r}(\mathbf{X}, \mathbf{Y})}{\partial x_{1} \partial x_{2} \ldots \partial x_{M_{1}} \partial y_{1} \partial y_{2} \ldots \partial y_{M_{2}}} \leqslant 0 \quad \forall x_{m_{1}} \in\left[x_{m_{1}}^{\langle s\rangle} ; x_{m_{1}}^{\langle s+1\rangle}\right] \quad \text { and } \\
\forall y_{m_{2}} \in\left[y_{m_{2}}^{\langle s\rangle} ; y_{m_{2}}^{\langle s+1\rangle}\right], \quad r \in\{1,2\}
\end{gathered}
$$

Of course, requirements (11) are very formal as they mean that any extremum of the player's payoff function, existing off the boundary of the hypercube (1), must be reached at points, which have only components

$$
\left\{\left\{\left\{x_{m_{1}}\langle s\rangle\right\}_{s=2}^{S_{1}\left(m_{1}\right)}\right\}_{m_{1}=1}^{M_{1}},\left\{\left\{y_{m_{2}}\langle\}_{s=2}\right\}_{2}\left(m_{2}\right)\right\}_{m_{2}=1}^{M_{2}}\right\}
$$

and nothing else. Such requirements can hardly be satisfied along a hypercube (1) dimension. They might have been met for the artificially retrofitted payoff functions $\left\{K_{r}(\mathbf{X}, \mathbf{Y})\right\}_{r \in\{1,2\}}$ before, though.

If the payoff functions $\left\{K_{r}(\mathbf{X}, \mathbf{Y})\right\}_{r \in\{1,2\}}$ cannot be retrofitted to requirements (11) then there are less severe requirements to the sampling. Shall the numbers (9) be chosen so that $\forall s=\overline{1, S_{r}\left(m_{r}\right)+1}$ by $m_{r}=\overline{1, M_{r}}$ there would be

$$
\begin{gathered}
\left|\frac{\partial^{M_{1}+M_{2}} K_{r}(\mathbf{X}, \mathbf{Y})}{\partial x_{1} \partial x_{2} \ldots \partial x_{M_{1}} \partial y_{1} \partial y_{2} \ldots \partial y_{M_{2}}}\right| \leqslant \alpha \quad \forall x_{m_{1}} \in\left[x_{m_{1}}^{\langle s\rangle} ; x_{m_{1}}^{\langle s+1\rangle}\right] \quad \text { and } \\
\forall y_{m_{2}} \in\left[y_{m_{2}}^{\langle s\rangle} ; y_{m_{2}}^{\langle s+1\rangle}\right], \quad r \in\{1,2\}
\end{gathered}
$$

Here in (12) the aggregate tolerable unsteadiness (ATU) of the players' payoff functions is denoted by the value $\alpha>0$, being the function of the range value

$$
\begin{aligned}
v_{\alpha} & =\max _{r \in\{1,2\}} \max _{\mathbf{X} \in U_{M_{1}}} \max _{\mathbf{Y} \in U_{M_{2}}} K_{r}(\mathbf{X}, \mathbf{Y})- \\
& -\min _{r \in\{1,2\}} \min _{\mathbf{X} \in U_{M_{1}}} \min _{\mathbf{Y} \in U_{M_{2}}} K_{r}(\mathbf{X}, \mathbf{Y}) .
\end{aligned}
$$


ATU is preset as a sufficiently small part of the range value (13). For instance, ATU may be conventionally preset to $\alpha \leqslant 0.01 \cdot v_{\alpha}$ or $\alpha \leqslant 0.005 \cdot v_{\alpha}$, depending on practical argumentations. Nevertheless, the satisfied requirements (12) are not the concluding step in converting the infinite game (3) into the bimatrix game. We should firstly arrange the finite game (6) by (7) into the bimatrix game. Secondly, acceptance of the drawn bimatrix game depends on features of its solution, approximating a solution of the infinite game (3).

\section{RESHAPING THE MULTIDIMENSIONAL MATRICES INTO TWO-DIMENSIONAL MATRICES}

After having $\left\{\left[S_{r}\left(m_{r}\right)\right]^{-1}\right\}_{m_{r}=1}^{M_{r}}$-sampled the hypersurface $K_{r}(\mathbf{X}, \mathbf{Y})$, the $r$-th player's payoff function has become $\left(M_{1}+M_{2}\right)$-dimensional matrix $\mathbf{P}_{r}(S(0))=\left[p_{J}^{\langle r\rangle}(S(0))\right]_{\mathscr{F}}$ by

$$
S(0)=\left\{\left\{S_{1}\left(m_{1}\right)\right\}_{m_{1}=1}^{M_{1}},\left\{S_{2}\left(m_{2}\right)\right\}_{m_{2}=1}^{M_{2}}\right\}
$$

of the format

$$
\mathscr{F}=\left(\chi_{m_{1}=1}^{M_{1}}\left(S_{1}\left(m_{1}\right)+1\right)\right) \times\left(\chi_{m_{2}=1}^{M_{2}}\left(S_{2}\left(m_{2}\right)+1\right)\right) .
$$

The matrix $\mathbf{P}_{r}(S(0))=\left[p_{J}^{\langle r\rangle}(S(0))\right]_{\mathscr{F}}$ elements

$$
\begin{gathered}
p_{J}^{\langle r\rangle}(S(0))=K_{r}(\mathbf{X}, \mathbf{Y}) \text { by } x_{m_{1}}=\frac{j_{m_{1}}-1}{S_{1}\left(m_{1}\right)} \\
\forall m_{1}=\overline{1, M_{1}} \text { and } y_{m_{2}}=\frac{j_{M_{1}+m_{2}}-1}{S_{2}\left(m_{2}\right)} \quad \forall m_{2}=\overline{1, M_{2}}
\end{gathered}
$$

have their indices

$$
\begin{gathered}
J=\left\{j_{k}\right\}_{k=1}^{M_{1}+M_{2}}, \quad j_{M_{1} \cdot \operatorname{sign}(r-1)+m_{r}} \in\left\{\overline{1, S_{r}\left(m_{r}\right)+1}\right\} \\
\forall m_{r}=\overline{1, M_{r}} \text { by } r \in\{1,2\} .
\end{gathered}
$$

Finite game (6) by (7) is solved outright as a bimatrix game if $M_{1}=M_{2}=1$. For $M_{1} \in \mathbb{N} \backslash\{1\}$ or $M_{2} \in \mathbb{N} \backslash\{1\}$ game (6) by (7) ought to be arranged into the bimatrix game through reshaping $\left(M_{1}+M_{2}\right)$-dimensional matrices $\mathbf{P}_{1}(S(0))$ and $\mathbf{P}_{2}(S(0))$ into ordinary two-dimensional matrices. The reverse index-to-index reshaping is also needed because the bimatrix game solution will be imposed on game (6) by (7).

Theorem 1. $\left(M_{1}+M_{2}\right)$-dimensional matrix

$$
\mathbf{P}_{r}(S(0))=\left[p_{J}^{\langle r\rangle}(S(0))\right]_{\mathscr{g}}
$$

of the format (14) with elements (15) is unambiguously reshaped into the ordinary flat matrix

$$
\mathbf{G}_{r}(S(0))=\left[g_{u_{1} u_{2}}^{\langle r\rangle}(S(0))\right]_{C F_{2}}
$$

of the format

$$
\mathscr{F}_{2}=\prod_{m_{1}=1}^{M_{1}}\left(S_{1}\left(m_{1}\right)+1\right) \times \prod_{m_{2}=1}^{M_{2}}\left(S_{2}\left(m_{2}\right)+1\right)
$$

with elements $g_{u_{1} u_{2}}^{\langle r\rangle}(S(0))=p_{J}^{\langle r\rangle}(S(0))$, using indices

$$
\begin{gathered}
u_{r}=\sum_{m_{r}=1}^{M_{r}}\left(\prod_{n=1}^{m_{r}-1}\left(S_{r}\left(M_{r}-n+1\right)+1\right)\right) \cdot\left(j_{M_{0}-m_{r}+1}-\operatorname{sign}\left(m_{r}-1\right)\right) \\
\text { at } M_{0}=\sum_{i=1}^{r} M_{i} \text { by } r \in\{1,2\}
\end{gathered}
$$

This reshaping is reversible.

Proof. It is obvious that for any set (16) of indices of the element $p_{J}^{\langle r\rangle}$ value (19) is integer and unique. Moreover, $u_{r} \in \mathbb{N}$ and, explicitly, $u_{r}=\overline{\prod_{m_{r}=1}^{M_{r}}\left(S_{r}\left(m_{r}\right)+1\right)}$ by $r \in\{1,2\}$. Thus, let the function $\psi(a, b)$ by $b \neq 0$ round the fraction $\frac{a}{b}$ to the nearest integer towards zero. And let

$$
\rho(a, b)=a-b \cdot \psi(a, b) .
$$

Then from integer (19) we get indices in set (16):

$$
\begin{aligned}
j_{M_{0}} & =\rho\left(u_{r}, S_{r}\left(M_{r}\right)+1\right)+\left(S_{r}\left(M_{r}\right)+1\right)\left(1-\operatorname{sign}\left[\rho\left(u_{r}, S_{r}\left(M_{r}\right)+1\right)\right]\right) \text { at } M_{0}=\sum_{i=1}^{r} M_{i} \text { and } \\
j_{M_{0}-m_{r}} & =1+\rho\left(\frac{u_{r}-j_{M_{0}}-\sum_{n=1}^{m_{r}-1}\left(\prod_{n_{1}=1}^{n}\left(S_{r}\left(M_{r}-n_{1}+1\right)+1\right)\right) \cdot\left(j_{M_{0}-n}-1\right)}{\prod_{n=1}^{m_{r}}\left(S_{r}\left(M_{r}-n+1\right)+1\right)}, S_{r}\left(M_{r}-m_{r}\right)+1\right) \forall m_{r}=\overline{1, M_{r}-1} .
\end{aligned}
$$


Therefore statements (19) and (20) define the reversible map of any set (16) into the set $\left\{u_{1}, u_{2}\right\}$. The theorem has been proven.

Theorem 1 allows considering finite game (6) by (7) as a bimatrix one. Therefore, let $z_{u_{r}}(S(0))$ be the $r$-th player's pure strategy, corresponding to its pure strategy in initial infinite game (3) with components in (15), whose indices are included into set (16). Hence, the first $M_{1}$ indices $\left\{j_{m_{1}}\right\}_{m_{1}=1}^{M_{1}}$ in set (16) correspond to components of the first player's pure strategy with its number $u_{1}$, and the last $M_{2}$ indices $\left\{j_{M_{1}+m_{2}}\right\}_{m_{2}=1}^{M_{2}}$ in set (16) correspond to components of the second player's pure strategy with its number $u_{2}$.

\section{INFINITE TWO-PERSON NON-COOPERATIVE GAME APPROXIMATION}

With the help of Theorem 1, finite game (6) by (7) is mapped into the bimatrix one

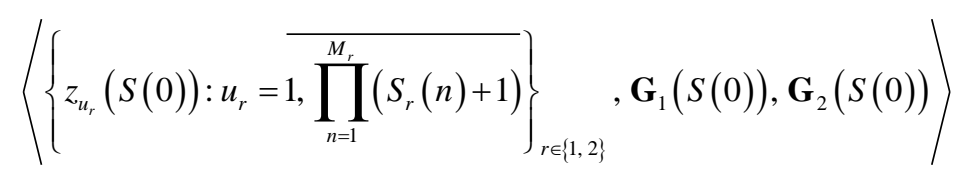

of the format (18) and inversely. Game (21), approximating game (3), has many solutions in forms of utility or equity equilibrium - Nash equilibrium, strong Nash equilibrium [7], [23], [31], [32], Pareto equilibrium [2], [6], [33], [34], Mertens-stable equilibrium [35], trembling hand perfect equilibrium [36], perfect Bayesian equilibrium [9], [37], [38], Markov perfect equilibrium [39], [40] and many others. For further action, let us denote by

$$
\begin{gathered}
\left\{\left\{q_{r}^{*}\left(z_{u_{r}}(S(0))\right)\right\}: u_{r}=1, \prod_{n=1}^{M_{r}}\left(S_{r}(n)+1\right)\right\} \begin{array}{l}
\text { (21), but what would be conditions of acceptance of such an } \\
\text { approximation? Naturally, the } r \text {-th player's payoff in } \\
\text { equilibrium situation }(22)
\end{array} \\
v_{r}(S(0))=\sum_{u_{1}=1}^{\pi\left(M_{1}, 0\right)} \sum_{u_{2}=1}^{\pi\left(M_{2}, 0\right)} g_{u_{1} u_{2}}^{\langle r\rangle}(S(0)) \cdot q_{1}^{*}\left(z_{u_{1}}(S(0))\right) \cdot q_{2}^{*}\left(z_{u_{2}}(S(0))\right)= \\
=\sum_{u_{1}^{*}(S(0)) \in Q_{1}^{*}(S(0))}\left(\sum_{u_{2}^{*}(S(0)) \in Q_{2}^{*}(S(0))} g_{u_{1}^{*}(S(0)) u_{2}^{*}(S(0))}^{\langle r\rangle}(S(0)) \cdot q_{1}^{*}\left(z_{u_{1}^{*}(S(0))}(S(0))\right) \cdot q_{2}^{*}\left(z_{u_{2}(S(0))}(S(0))\right)\right), r \in\{1,2\},
\end{gathered}
$$

one of those solution types, where $q_{r}^{*}\left(z_{u_{r}}(S(0))\right)$ is probability of applying the pure strategy $z_{u_{r}}(S(0))$ by the $r$-th player, letting at $u_{r}=1, \overline{\prod_{n=1}^{M_{r}}\left(S_{r}(n)+1\right)}$ reach the determinate equilibrium. It might seem that game (3) had been approximately solved using solution (22) of bimatrix game

where

$$
\begin{gathered}
\pi\left(M_{r}, 0\right)=\prod_{n=1}^{M_{r}}\left(S_{r}(n)+1\right), \\
Q_{r}^{*}(S(0))=\left\{u_{r}^{*}(S(0)) \in\left\{\overline{1, \pi\left(M_{r}, 0\right)}\right\}: q_{r}^{*}\left(z_{u_{r}^{*}(S(0))}(S(0))\right)>0, q_{r}^{*}\left(z_{u_{r}}(S(0))\right)=0 \forall u_{r} \notin Q_{r}^{*}(S(0))\right\},
\end{gathered}
$$

is not a factor of the acceptance, because the players' genuine payoffs in game (3), taken by the situation whose approximation is (22), may be unknown. Henceforward, the infinite two-person non-cooperative game approximation (21) with its solution (22) should be studied whether the $r$-th player's ESS (regarding the corresponding probabilities)

$$
\left\{q_{r}^{*}\left(z_{u_{r}^{*}(S(0))}(S(0))\right)\right\}_{u_{r}^{*}(S(0)) \in Q_{r}^{*}(S(0))}
$$

is consistent to other supports under

$$
\begin{gathered}
S(\lambda)=\left\{\left\{S_{1}\left(m_{1}\right)+\lambda\right\}_{m_{1}=1}^{M_{1}},\left\{S_{2}\left(m_{2}\right)+\lambda\right\}_{m_{2}=1}^{M_{2}}\right\} \\
\text { by } \lambda \in \mathbb{Z} \backslash\{0\} .
\end{gathered}
$$

The $r$-th player's payoff (23) shall be subjoined, too.

\section{CONSISTENCY OF THE PLAYER'S ESS}

Apparently, there is nothing except two sets (24) for $r \in\{1,2\}$ generating the payoffs $\left\{v_{r}(S(0))\right\}_{r \in\{1,2\}}$ by (23) to pretend to the game approximation acceptance. The question is, what the approximation would be by a lesser sampling step. 
To be stricter, it is rather "should" than "would", though: the accepted approximation must have properties that will not be confused under more scrupulous approximations.

For every specified sampling step, being determined with integers (9), its minimal neighbourhood can be considered. Due to denotation (25), this neighbourhood is determined with sets $\{S(1), S(-1)\}$ of integers. And for the first priority, the game approximation is acceptable if the players' payoffs in the bimatrix game change no more by decreasing minimally the sampling steps along every dimension of unit hypercube (1) than if the sampling steps were increased minimally:

$$
\begin{gathered}
\left|v_{r}(S(0))-v_{r}(S(1))\right| \leqslant \\
\leqslant\left|v_{r}(S(-1))-v_{r}(S(0))\right|, \quad r \in\{1,2\},
\end{gathered}
$$

where

$$
\begin{aligned}
& v_{r}(S(\lambda))=\sum_{u_{1}=1}^{\pi\left(M_{1}, \lambda\right)} \sum_{u_{2}=1}^{\pi\left(M_{2}, \lambda\right)} g_{u_{1} u_{2}}^{\langle r\rangle}(S(\lambda)) \cdot q_{1}^{*}\left(z_{u_{1}}(S(\lambda))\right) \cdot q_{2}^{*}\left(z_{u_{2}}(S(\lambda))\right)= \\
& =\sum_{u_{1}^{*}(S(\lambda)) \in Q_{1}^{*}(S(\lambda))}\left(\sum_{u_{2}^{*}(S(\lambda)) \in Q_{2}^{*}(S(\lambda))} g_{u_{1}^{*}(S(\lambda)) u_{2}^{*}(S(\lambda))}^{\langle r\rangle}(S(\lambda)) \cdot q_{1}^{*}\left(z_{u_{1}^{*}(S(\lambda))}(S(\lambda))\right) \cdot q_{2}^{*}\left(z_{u_{2}^{*}(S(\lambda))}(S(\lambda))\right)\right) \text {, } \\
& \pi\left(M_{r}, \lambda\right)=\prod_{n=1}^{M_{r}}\left(S_{r}(n)+1+\lambda\right) \\
& Q_{r}^{*}(S(\lambda))=\left\{u_{r}^{*}(S(\lambda)) \in\left\{\overline{1, \pi\left(M_{r}, \lambda\right)}\right\}: q_{r}^{*}\left(z_{u_{r}^{*}(S(\lambda))}(S(\lambda))\right)>0, q_{r}^{*}\left(z_{u_{r}}(S(\lambda))\right)=0 \forall u_{r} \notin Q_{r}^{*}(S(\lambda))\right\} \text {. }
\end{aligned}
$$

Furthermore, with the minimal decrement of the sampling steps the cardinality of ESS (24) shall not decrease:

$$
\left|Q_{r}^{*}(S(1))\right| \geqslant\left|Q_{r}^{*}(S(0))\right| \text { by } r \in\{1,2\} \text {. }
$$

Notwithstanding exclusive importance of (26) and (27) for the game approximation acceptance, ESS (24) may change heavily in comparison with

$$
\left\{q_{r}^{*}\left(z_{u_{r}^{*}(S(1))}(S(1))\right)\right\}_{u_{r}^{*}(S(1)) \in Q_{r}^{*}(S(1))}
$$

or with (28) and

$$
\left\{q_{r}^{*}\left(z_{u_{r}^{*}(S(-1))}(S(-1))\right)\right\}_{u_{r}^{*}(S(-1)) \in Q_{r}^{*}(S(-1))}
$$

jointly. Thus, acceptance of game (21) solution as an approximate solution of game (3) necessitates a sufficient closeness of the player's ESS, being obtained within minimal neighbourhood of the sampling steps. For the closeness consideration we need to regard a piecewise linear hypersurface $h_{r}\left(u_{r}, S(0)\right)$ whose vertices are in points

$$
\left\{\left\{\frac{j_{M_{1} \cdot \operatorname{sign}(r-1)+m_{r}}-1}{S_{r}\left(m_{r}\right)}\right\}_{m_{r}=1}^{M_{r}}, q_{r}^{*}\left(z_{u_{r}}(S(0))\right)\right\}
$$

in $\mathbb{R}^{M_{p}+1}$ for $r \in\{1,2\}$. Additionally we will mark out the nonzero vertices among (30) as points of hypercube $U_{M_{r}}$, matching the set $Q_{r}^{*}(S(0))$. For this reason, having unrolled every index $u_{r}^{*}(S(0)) \in Q_{r}^{*}(S(0))$ by (20) back to indices' set $\left.\left\{j_{m_{r}} u_{r}^{*}(S(0))\right\rangle\right\}_{m_{r}=1}^{M_{r}}$, the index $u_{1}^{*}(S(0)) \in Q_{1}^{*}(S(0))$ is matched to the point

$$
\begin{gathered}
\mathbf{X}_{u_{1}^{*}(S(0))}=\left[x_{m_{1}}^{\left\langle u_{1}^{*}(S(0))\right\rangle}\right]_{1 \times M_{1}}=\left[\frac{j_{m_{1}}^{\left\langle u_{1}^{*}(S(0))\right\rangle}-1}{S_{1}\left(m_{1}\right)}\right]_{1 \times M_{1}} \in U_{M_{1}} \\
\forall u_{1}^{*}(S(0)) \in Q_{1}^{*}(S(0)),
\end{gathered}
$$

and the index $u_{2}^{*}(S(0)) \in Q_{2}^{*}(S(0))$ is matched to the point

$$
\begin{gathered}
\mathbf{Y}_{u_{2}^{*}(S(0))}=\left[y_{m_{2}}^{\left\langle u_{2}^{*}(S(0))\right\rangle}\right]_{1 \times M_{2}}=\left[\frac{j_{M_{1}+m_{2}}^{\left\langle u_{2}^{*}(S(0))\right\rangle}-1}{S_{2}\left(m_{2}\right)}\right]_{1 \times M_{2}} \in U_{M_{2}} \\
\forall u_{2}^{*}(S(0)) \in Q_{2}^{*}(S(0)) .
\end{gathered}
$$

Furthermore, let the points $\left\{\mathbf{X}_{u_{1}^{*}(S(0))}\right\}_{u_{1}^{*}(S(0)) \in Q_{1}^{*}(S(0))}$ and $\left\{\mathbf{Y}_{u_{2}^{*}(S(0))}\right\}_{u_{2}^{*}(S(0)) \in Q_{2}^{*}(S(0))}$ be sorted into sets

$$
\begin{gathered}
\left\{\overline{\mathbf{X}}_{u_{1}^{*}(S(0))}\right\}_{u_{1}^{*}(S(0)) \in Q_{1}^{*}(S(0))}=\left\{\left[\frac{\bar{j}_{m_{1}}^{\left.u_{1}^{*}(S(0))\right\rangle}-1}{S_{1}\left(m_{1}\right)}\right]_{1 \times M_{1}}\right\}_{u_{1}^{*}(S(0)) \in Q_{1}^{*}(S(0))}= \\
=\left\{\mathbf{X}_{u_{1}^{*}(S(0))}\right\}_{u_{1}^{*}(S(0)) \in Q_{1}^{*}(S(0))}
\end{gathered}
$$


and

$$
\begin{gathered}
\left\{\overline{\mathbf{Y}}_{u_{2}^{*}(S(0))}\right\}_{u_{2}^{*}(S(0)) \in Q_{2}^{*}(S(0))}=\left\{\left[\frac{\bar{j}_{M_{1}+m_{2}}^{\left\langle\left\langle u^{*}(S(0))\right.\right.}-1}{S_{2}\left(m_{2}\right)}\right]_{1 \times M_{2}}\right\}_{u_{2}^{*}(S(0)) \in Q_{2}^{*}(S(0))}= \\
=\left\{\mathbf{Y}_{u_{2}^{*}(S(0))}\right\}_{u_{2}^{*}(S(0)) \in Q_{2}^{*}(S(0))}
\end{gathered}
$$

that the value

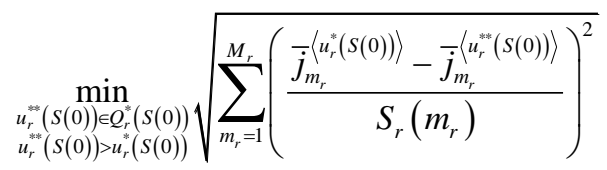

is reached at

$$
\begin{gathered}
u_{r}^{* *}(S(0)) \in \arg \min _{\substack{u_{r}^{* *} \\
\text { und } \\
u_{r}^{* *}(S(0)) \in Q_{r}^{*}(S(0))>u_{r}^{*}(S(0))}}\left(u_{r}^{* * * * *}(S(0))-u_{r}^{*}(S(0))\right) \\
\forall u_{r}^{*}(S(0)) \in Q_{r}^{*}(S(0)) \backslash\left\{\max \left(Q_{r}^{*}(S(0))\right)\right\}
\end{gathered}
$$

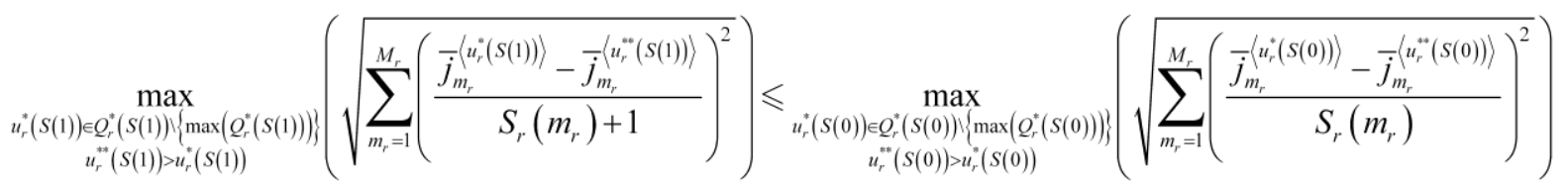

at

$$
\begin{gathered}
u_{r}^{* *}(S(1)) \in \arg \min _{\substack{u_{r}^{* * *}(S(1)) \in Q_{r}^{*}(S(1)) \\
u_{r}^{* *}(S(1))>u_{r}^{*}(S(1))}}\left(u_{r}^{* * * * *}(S(1))-u_{r}^{*}(S(1))\right) \\
\forall u_{r}^{*}(S(1)) \in Q_{r}^{*}(S(1)) \backslash\left\{\max \left(Q_{r}^{*}(S(1))\right)\right\}
\end{gathered}
$$

and (31).

Definition 1. Solution (22) of game (21) is called weakly consistent under set $S(0)$ for being game (3) approximate solution if there are true inequalities (26), (27), and (32)-(34) at (35), (31) by $r \in\{1,2\}$. If these inequalities are true for a player's ESS then this player's ESS or the strategy itself is

by $r \in\{1,2\}$. Thus, the game approximation is acceptable if the players' ESS piecewise linear hypersurfaces change no more by decreasing minimally the sampling steps along every dimension of the unit hypercube (1) than if the sampling steps were increased minimally:

$$
\begin{aligned}
& \max _{U_{M_{r}}}\left|h_{r}\left(u_{r}, S(0)\right)-h_{r}\left(u_{r}, S(1)\right)\right| \leqslant \\
& \leqslant \max _{U_{M_{r}}}\left|h_{r}\left(u_{r}, S(-1)\right)-h_{r}\left(u_{r}, S(0)\right)\right|
\end{aligned}
$$

and

$$
\begin{gathered}
\left\|h_{r}\left(u_{r}, S(0)\right)-h_{r}\left(u_{r}, S(1)\right)\right\| \leqslant \\
\leqslant\left\|h_{r}\left(u_{r}, S(-1)\right)-h_{r}\left(u_{r}, S(0)\right)\right\| \text { in } \mathbb{L}_{2}\left(U_{M_{r}}\right)
\end{gathered}
$$

by $r \in\{1,2\}$. With the minimal decrement of the sampling steps into $S(1)$ there should not be decrement in density of points with nonzero probabilities of their selection on the hypercube (2):

called weakly consistent under set $S(0)$.

This is a definition of the most primitive consistency for the approximate solution of game (3). It could be strengthened in (27) and (34) with the part of $S(-1)$, which would make the cardinality of every player's ESS and their densities be non-decreasing within minimal neighbourhood of the sampling steps.

Definition 2. Weakly consistent under set $S(0)$ solution (22) of game (21) is called consistent for being game (3) approximate solution if

$$
\left|Q_{r}^{*}(S(0))\right| \geqslant\left|Q_{r}^{*}(S(-1))\right|,
$$

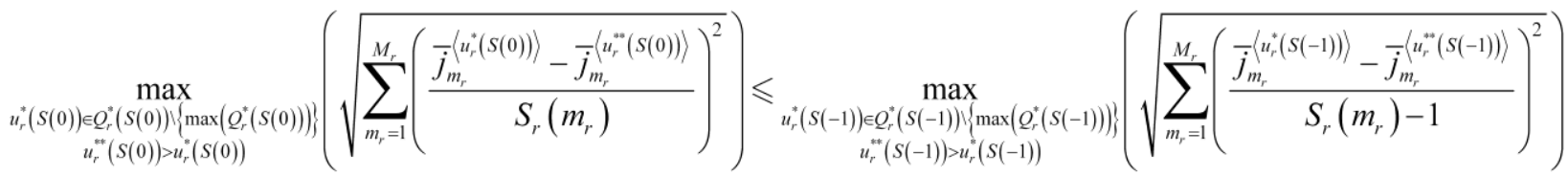

at (31) and

$$
u_{r}^{* * *}(S(-1)) \in \arg \min _{\substack{u_{r}^{-*}(S(-1)) \in Q_{r}^{*}(S(-1)) \\ \text { unt } \\ u_{r}^{* *}(S(-1))>u_{r}^{*}(S(-1))}}\left(u_{r}^{* * * * *}(S(-1))-u_{r}^{*}(S(-1))\right)
$$

by $r \in\{1,2\}$. If these inequalities are true for a player's itself is called consistent under set $S(0)$. 
Clearly, controlling $S(0)$-consistency requires more computational resources than controlling weak $S(0)-$ consistency. Therefore, it is senseless to check whether ESS is consistent or not, if a researcher is not sure about its weakly $S(0)$-consistency.

Theorem 2. If in the game

$$
\left\langle\left\{z_{u_{r}}(S(1)): u_{r}=1, \prod_{n=1}^{M_{r}}\left(S_{r}(n)+2\right)\right\}_{r \in\{1,2\}}, \mathbf{G}_{1}(S(1)), \mathbf{G}_{2}(S(1))\right\rangle
$$

there is a completely mixed Nash equilibrium situation, then for checking the weak $S(0)$-consistency of the Nash equilibrium situation in game (21) it is sufficient to check inequalities (26), (32), (33).

Proof. Inasmuch as the Nash equilibrium situation is completely mixed then

$$
\begin{aligned}
& \left|Q_{r}^{*}(S(1))\right|=\pi\left(M_{r}, 1\right)=\prod_{n=1}^{M_{r}}\left(S_{r}(n)+2\right) \geqslant \\
& \geqslant \prod_{n=1}^{M_{r}}\left(S_{r}(n)+1\right)=\pi\left(M_{r}, 0\right)=\left|Q_{r}^{*}(S(0))\right|,
\end{aligned}
$$

that gives us (27). At the same time, density of all the points of lattices

$$
\chi_{m_{1}=1}^{M_{1}} D_{m_{1}}^{\langle\mathbf{x}\rangle}\left(S_{1}\left(m_{1}\right)+1\right)
$$

and

$$
\chi_{m_{2}=1}^{M_{2}} D_{m_{2}}^{\langle\mathbf{Y}\rangle}\left(S_{2}\left(m_{2}\right)+1\right)
$$

is greater than density of all the points of sparser lattices

$$
\chi_{m_{1}=1}^{M_{1}} D_{m_{1}}^{\langle\mathbf{X}\rangle}\left(S_{1}\left(m_{1}\right)\right)
$$

and

$$
\sum_{m_{2}=1}^{M_{2}} D_{m_{2}}^{\langle\mathbf{Y}\rangle}\left(S_{2}\left(m_{2}\right)\right)
$$

that confirms (34) at (31) and (35). The theorem has been proved.

Remarkably that the completely mixed Nash equilibrium situation in game (39) is unique by

$$
\prod_{m_{1}=1}^{M_{1}}\left(S_{1}\left(m_{1}\right)+2\right)=\prod_{m_{2}=1}^{M_{2}}\left(S_{2}\left(m_{2}\right)+2\right)=N
$$

and $\operatorname{det} \mathbf{G}_{1}(S(1)) \neq 0, \operatorname{det} \mathbf{G}_{2}(S(1)) \neq 0$, where [30]

$$
\begin{aligned}
& v_{r}(S(1))=\frac{1}{\mathbf{I}_{N} \cdot\left[\mathbf{G}_{r}(S(1))\right]^{-1} \cdot \mathbf{I}_{N}^{\mathrm{T}}} \text { by } r \in\{1,2\} \\
& \mathbf{Q}_{1}^{*}(S(1))=\left[q_{1}^{*}\left(z_{u_{1}}(S(1))\right)\right]_{1 \times N}= \\
&=v_{2}(S(1)) \cdot \mathbf{I}_{N} \cdot\left[\mathbf{G}_{2}(S(1))\right]^{-1} \\
& \mathbf{Q}_{2}^{*}(S(1))=\left[q_{2}^{*}\left(z_{u_{2}}(S(1))\right)\right]_{1 \times N}= \\
&=v_{1}(S(1)) \cdot\left(\left[\mathbf{G}_{1}(S(1))\right]^{-1} \cdot \mathbf{I}_{N}^{\mathrm{T}}\right)^{\mathrm{T}}
\end{aligned}
$$

that speeds up checking the weak $S(0)$-consistency of the Nash equilibrium situation. Consistent solution (22) is nonetheless more confident for game (3) approximation than weak consistent solution (22). Weak $S(0)$-consistent solution (22) is at least an argument for its acceptance in game (3) approximation.

\section{DISCUSSING THE ACCEPTABILITY OF INFINITE TWO-PERSON GAME APPROXIMATION}

Finding Nash equilibria in an infinite game is not commonly computable. Therefore, any approach to solve such games is considered a valuable contribution to the game theory and its practical applications. The solution will be approximate, but the rate of approximateness is adjustable owing to sampling. Consistency of the solution and its conditions are criteria of approximability. If game (3) is approximable then it is acceptable.

Note that the limit

$$
\lim _{\lambda \rightarrow \infty} v_{r}(S(\lambda))
$$

existence is still non-asserted. In general, it has not been proven yet that (weak) consistency of the $r$-th player's ESS is necessarily followed by that limit

$$
\lim _{\lambda \rightarrow \infty} h_{r}\left(u_{r}, S(\lambda)\right) .
$$

Even though limit (45) exists, we do not know how close it is (in sense of metric in the corresponding functional space) to the $r$-th player's genuine equilibrium strategy in game (3). 
Similarly, if there is limit (44) there is no certainty about that this limit is the $r$-th player's genuine payoff in game (3), taken by the suggested equilibrium situation whose approximation is (22). Moreover, any consistency of the player's ESS is incomprehensible whether it involves the other player's ESS into any consistency. Nevertheless, these lacks do not depreciate that the defined consistency helps reveal natural properties of infinite two-person game approximation. These properties include bounded variation of payoff, of ESS cardinality and its density, functional closeness of ESS by (32) and (33).

In fact, the case when

$$
\prod_{m_{1}=1}^{M_{1}}\left(S_{1}\left(m_{1}\right)+1\right)=\prod_{m_{2}=1}^{M_{2}}\left(S_{2}\left(m_{2}\right)+1\right)
$$

and $\operatorname{det} \mathbf{G}_{1}(S(0)) \neq 0, \operatorname{det} \mathbf{G}_{2}(S(0)) \neq 0$, is very favourable for solving bimatrix game (21) in one statement [30]. In this case, numbers (9) should be adjusted at (46). A version of how to do that is taking

$$
S_{2}\left(m_{2}\right)=S_{0} \quad \forall m_{2}=\overline{1, M_{2}}
$$

and

$$
\begin{aligned}
& S_{1}\left(m_{1}\right)=S_{0} \quad \forall m_{1}=\overline{1, M_{1}-1} \\
& \text { at } S_{1}\left(M_{1}\right)=\left(S_{0}+1\right)^{M_{2}-M_{1}+1}-1
\end{aligned}
$$

for $M_{1}<M_{2}$.

Eventually, the stated consistency concept is possible to expand on any form of equilibrium of utility or equity equilibrium. However, the consistency result of these forms will be different. If solution (22) appears non-consistent even weakly, then numbers (9) of intervals between the selected points on the hypercube (1) should be increased. Note, only one or a few of those numbers (not all) could be increased, and that might make the solution consistent. This question needs a separate investigation, as well as the question of ability of the consistency to become disappeared after increments within a subset of numbers (9).

\section{CONCLUSION}

The proposed concept of the solution consistency is a necessary condition to regard acceptability of an infinite twoperson game approximation. The sufficiency is connected with unproved limits (44) and (45). First of all, it is necessary to check the tolerable closeness of ESS for both players when numbers (9) are changed minimally. Subsequently, the consistent solution certifies the game approximation acceptability, enabling one to solve even games without any equilibrium situations [2], [30], [41], [42], including isomorphic ones to the non-cooperative game on unit hypercube of EFDS.

The suggested sampling for solving approximately the infinite two-person game (3) on unit hypercube (1) is fulfilled in three stages. Primarily, payoff functions $\left\{K_{r}(\mathbf{X}, \mathbf{Y})\right\}_{r \in\{1,2\}}$ are sampled by sets (9) according to requirements (11) or (12). Then, having obtained sets of points (4) and (5), $\left(M_{1}+M_{2}\right)-$ dimensional matrices $\left\{\mathbf{P}_{r}(S(0))\right\}_{r \in\{1,2\}}$ with their elements (15) and indices (16) are reshaped into ordinary flat matrices (17) of format (18), using new indices (19). At the third stage, game (21) solution (22) is checked out for its weak consistency by inequalities (26), (27), and (32)-(34) at (35) and (31) by $r \in\{1,2\}$. If it is necessary, weakly consistent solution (22) is checked out for its consistency by inequalities (36) and (37) at (31) and (38) by $r \in\{1,2\}$.

An unclosed item of solution consistency is a possibility to determine (weak) consistency of the player's ESS when the other player's ESS consistency (maybe, weak) has been already determined. And shall one player use its (weakly) consistent ESS while the other player's ESS is not consistent (or just weakly consistent)? These questions motivate further contribution to sampling and approximating the infinite twoperson non-cooperative games in EFDS. Furthermore, generalisation in sampling (open and semi-open) hypercubes $U_{M_{1}}$ and $U_{M_{2}}$ non-uniformly is going to be stated. The furthermost vista is to state "wider" consistency concept, entailing sets $\{S(\lambda)\}_{\lambda \in \mathbb{Z}}$ for $|\lambda| \leqslant L \in \mathbb{N} \backslash\{1\}$. However, it will be anyway impossible to sweep the players' ESS in a very wide range of numbers (9). That is why the stated study has been restricted to $L=1$.

\section{REFERENCES}

[1] M. J. Osborne, An introduction to game theory. Oxford: Oxford University Press, 2003

[2] N. N. Vorobyov, Game theory fundamentals. Noncooperative games. Moscow: Nauka, 1984 (in Russian).

[3] J. Bergin, "A characterization of sequential equilibrium strategies in infinitely repeated incomplete information games," Journal of Economic Theory, vol. 47, iss. 1, pp. 51-65, Feb. 1989. https://doi.org/10.1016/0022-0531(89)90102-6

[4] B. Chen and S. Takahashi, "A folk theorem for repeated games with unequal discounting," Games and Economic Behavior, vol. 76, iss. 2, pp. 571-581, Nov. 2012. https://doi.org/10.1016/j.geb.2012.07.011

[5] V. V. Romanuke, "Method of practicing the optimal mixed strategy with innumerable set in its spectrum by unknown number of plays," Measuring and Computing Devices in Technological Processes, no. 2, pp. 196-203, 2008.

[6] P. V. Reddy and J. C. Engwerda, "Pareto optimality in infinite horizon linear quadratic differential games," Automatica, vol. 49, iss. 6, pp. 1705-1714, June 2013 https://doi.org/10.1016/j.automatica.2013.03.004

[7] X. Chen and X. Deng, "Recent development in computational complexity characterization of Nash equilibrium," Computer Science Review, vol. 1, iss. 2, pp. 88-99, Dec. 2007. https://doi.org/10.1016/j.cosrev.2007.09.002

[8] D. Friedman, "On economic applications of evolutionary game theory," Journal of Evolutionary Economics, vol. 8, iss. 1, pp. 15-43, March 1998. https://doi.org/10.1007/s001910050054

[9] S. Brusco, "Perfect Bayesian implementation in economic environments," Journal of Economic Theory, vol. 129, iss. 1, pp. 1-30, July 2006. https://doi.org/10.1016/j.jet.2004.12.002

[10] R. Calvert, M. D. McCubbins, and B. R. Weingast, "A Theory of Political Control and Agency Discretion," American Journal of Political Science, vol. 33, iss. 3, pp. 588-611, Aug. 1989.

https://doi.org/10.2307/2111064 
[11] H. Xie and Y.-J. Lee, "Social norms and trust among strangers," Games and Economic Behavior, vol. 76, iss. 2, pp. 548-555, Nov. 2012 https://doi.org/10.1016/j.geb.2012.07.010

[12] L. Forni, "Social security as Markov equilibrium in OLG models," Review of Economic Dynamics, vol. 8, iss. 1, pp. 178-194, Jan. 2005. https://doi.org/10.1016/j.red.2004.10.003

[13] R. Zhao, G. Neighbour, J. Han, M. McGuire, and P. Deutz, "Using game theory to describe strategy selection for environmental risk and carbon emissions reduction in the green supply chain," Journal of Loss Prevention in the Process Industries, vol. 25, iss. 6, pp. 927-936, Nov. 2012. https://doi.org/10.1016/j.jlp.2012.05.004

[14] J. Scheffran, "The dynamic interaction between economy and ecology: Cooperation, stability and sustainability for a dynamic-game model of resource conflicts," Mathematics and Computers in Simulation, vol. 53, iss. $4-6$, pp. $371-380$, Oct. 2000 https://doi.org/10.1016/S0378-4754(00)00229-9

[15] M. Braham and F. Steffen, "Voting rules in insolvency law: a simplegame theoretic approach," International Review of Law and Economics, vol. 22 , iss. 4 , pp. 421-442, Dec. 2002. https://doi.org/10.1016/S0144-8188(02)00113-8

[16] G. Stoltz and G. Lugosi, "Learning correlated equilibria in games with compact sets of strategies," Games and Economic Behavior, vol. 59, iss. 1, pp. 187-208, Apr. 2007. https://doi.org/10.1016/i.geb.2006.04.007

[17] E. Bajoori, J. Flesch, and D. Vermeulen, "Perfect equilibrium in games with compact action spaces," Games and Economic Behavior, vol. 82 , pp. 490-502, Nov. 2013. https://doi.org/10.1016/j.geb.2013.08.002

[18] J. Gabarró, A. García, and M. Serna, "The complexity of game isomorphism," Theoretical Computer Science, vol. 412, iss. 48 , pp. 6675-6695, Nov. 2011. https://doi.org/10.1016/j.tcs.2011.07.022

[19] C. E. Lemke and J. T. Howson, "Equilibrium points of bimatrix games," SIAM Journal on Applied Mathematics, vol. 12, iss. 2, pp. 413 423, 1964. https://doi.org/10.1137/0112033

[20] N. Nisan, T. Roughgarden, É. Tardos and V. V. Vazirani, eds., Algorithmic Game Theory. Cambridge, UK: Cambridge University Press, 2007. https://doi.org/10.1017/CBO9780511800481

[21] N. N. Vorobyov, "Situations of equilibrium in bimatrix games," Probability theory and its applications, no. 3, pp. 318-331, 1958 (in Russian).

[22] H. W. Kuhn, "An algorithm for equilibrium points in bimatrix games," Proceedings of the National Academy of Sciences of the United States of America, vol. 47, pp. 1657-1662, 1961. https://doi.org/10.1073/pnas.47.10.1657

[23] S. C. Kontogiannis, P. N. Panagopoulou and P. G. Spirakis, "Polynomial algorithms for approximating Nash equilibria of bimatrix games," Theoretical Computer Science, vol. 410, iss. 15, pp. 1599-1606, Apr. 2009. https://doi.org/10.1016/j.tcs.2008.12.033

[24] P. J. Reny, "On the Existence of Pure and Mixed Strategy Equilibria in Discontinuous Games," Econometrica, vol. 67, iss. 5, pp. 1029-1056, Sep. 1999. https://doi.org/10.1111/1468-0262.00069

[25] P. Bernhard and J. Shinar, "On finite approximation of a game solution with mixed strategies," Applied Mathematics Letters, vol. 3, iss. 1, pp. 1-4, 1990. https://doi.org/10.1016/0893-9659(90)90054-F

[26] S. C. Kontogiannis and P. G. Spirakis, "On mutual concavity and strategically-zero-sum bimatrix games," Theoretical Computer Science, vol. 432, pp. 64-76, May 2012. https://doi.org/10.1016/j.tcs.2012.01.016

[27] D. Friedman and D. N. Ostrov, "Evolutionary dynamics over continuous action spaces for population games that arise from symmetric two-player games," Journal of Economic Theory, vol. 148, iss. 2, pp. 743-777, March 2013. https://doi.org/10.1016/i.jet.2012.07.004

[28] R. Laraki, A. P. Maitra, and W. D. Sudderth, "Two-Person Zero-Sum Stochastic Games with Semicontinuous Payoff," Dynamic Games and Applications, vol. 3, iss. 2, pp. 162-171, June 2013. https://doi.org/10.1007/s13235-012-0054-7

[29] R. Gu, X. Yang, J. Yan, Y. Sun, B. Wang, C. Yuan and Y. Huang, "SHadoop: Improving MapReduce performance by optimizing job execution mechanism in Hadoop clusters," Journal of Parallel and Distributed Computing, vol. 74, iss. 3, pp. 2166-2179, March 2014 https://doi.org/10.1016/j.jpdc.2013.10.003
[30] H. Moulin, Théorie des jeux pour l'économie et la politique. Paris: Hermann, 1981 (in French).

[31] G. Tian, "Implementation of balanced linear cost share equilibrium solution in Nash and strong Nash equilibria," Journal of Public Economics, vol. 76, iss. 2, pp. 239-261, May 2000. https://doi.org/10.1016/S0047-2727(99)00041-9

[32] S.-C. Suh, "An algorithm for verifying double implementability in Nash and strong Nash equilibria," Mathematical Social Sciences, vol. 41, iss. 1, pp. 103-110, Jan. 2001 https://doi.org/10.1016/S0165-4896(99)00057-8

[33] V. Scalzo, "Pareto efficient Nash equilibria in discontinuous games," Economics Letters, vol. 107, iss. 3, pp. 364-365, 2010. https://doi.org/10.1016/j.econlet.2010.03.010

[34] D. Gąsior and M. Drwal, "Pareto-optimal Nash equilibrium in capacity allocation game for self-managed networks," Computer Networks, vol. 57 , iss. 14 , pp. $2817-2832$, Oct. 2013. https://doi.org/10.1016/i.comnet.2013.06.012

[35] E. Kohlberg and J.-F. Mertens, "On the Strategic Stability of Equilibria," Econometrica, vol. 54, no. 5, pp. 1003-1037, 1986. https://doi.org/10.2307/1912320

[36] R. Selten, "Reexamination of the perfectness concept for equilibrium points in extensive games," International Journal of Game Theory, vol. 4, iss. 1, pp. 25-55, March 1975. https://doi.org/10.1007/BF01766400

[37] E. van Damme, "A relation between perfect equilibria in extensive form games and proper equilibria in normal form games," International Journal of Game Theory, vol. 13, iss. 1, pp. 1-13, March 1984. https://doi.org/10.1007/BF01769861

[38] D. Fudenberg and J. Tirole, "Perfect Bayesian equilibrium and sequential equilibrium," Journal of Economic Theory, vol. 53, iss. 2, pp. 236-260, Apr. 1991 https://doi.org/10.1016/0022-0531(91)90155-W

[39] S. Castro and A. Brandão, "Existence of a Markov perfect equilibrium in a third market model," Economics Letters, vol. 66, iss. 3, pp. 297-301, March 2000. https://doi.org/10.1016/S0165-1765(99)00208-6

[40] H. Haller and R. Lagunoff, "Markov Perfect equilibria in repeated asynchronous choice games," Journal of Mathematical Economics, vol. 46, iss. 6, pp. 1103-1114, Nov. 2010. https://doi.org/10.1016/j.jmateco.2009.09.003

[41] K. Tanaka and K. Yokoyama, "On $\varepsilon$-equilibrium point in a noncooperative n-person game," Journal of Mathematical Analysis and Applications, vol. 160, iss. 2, pp. 413-423, Sep. 1991. https://doi.org/10.1016/0022-247X(91)90314-P

[42] T. Radzik, "Pure-strategy $\varepsilon$-Nash equilibrium in two-person non-zerosum games," Games and Economic Behavior, vol. 3, iss. 3, pp. 356-367, Aug. 1991. https://doi.org/10.1016/0899-8256(91)90034-C

Vadim V. Romanuke was born in 1979. In 2006, he received the Degree of Candidate of Technical Sciences in Mathematical Modelling and Computational Methods. The Degree of Doctor of Technical Sciences in Mathematical Modelling and Computational Methods was received in 2014.

$\mathrm{He}$ is a Professor of Applied Mathematics and Social Informatics Department at Khmelnitskiy National University, Ukraine. His research interests concern decision making, game theory, statistical approximation, and control engineering based on statistical correspondence.

Address for correspondence: Institutskaya Str., 11, 29016, Khmelnitskiy, Ukraine.

E-mail: romanukevadimv@mail.ru

Vladimir V. Kamburg was born in 1948. In 1999, he received the Degree of Doctor of Technical Sciences in Machines and Devices of Chemical Manufacturing.

$\mathrm{He}$ is a Professor of Information and Computing Systems Department at Penza State University of Architecture and Building, Russia. His research interests concern mathematical modelling of physical, environmental, and social processes, inverse problems, and game theory.

Address for correspondence: G. Titov Str., 28, 440028, Penza, Russia.

E-mail: kamburg@rambler.ru 